\title{
EMERGENCY SERVICE RESPONSE PLAN FOR COVID-19 PANDEMIC IN RESOURCE LIMITED SETTINGS
}

\author{
Ramesh Kumar Maharjan ${ }^{1^{*}}$, Jukel Shrestha ${ }^{2}$, Yogendra Man \\ Shakya ${ }^{3}$, Ramesh Prasad Aacharya ${ }^{4}$, Pratap Narayan Prasad \\ ${ }^{I}$ *Dr. Ramesh Kumar Maharjan, Associate Professor, MD GP, DM Emergency Medicine \\ ${ }^{2}$ Dr. Jukel Shrestha, MD GP, DM Emergency Medicine Resident \\ ${ }^{3}$ Prof. Dr. Yogendra Man Shakya, MD GP, Head of Department \\ ${ }^{4}$ Prof. Dr. Ramesh Prasad Aacharya, MD GP, MMEthic \\ ${ }^{5}$ Prof. Dr. Pratap Narayan Prasad, MD GP, Chief, MMC, IOM, TU \\ Department of General Practice and Emergency Medicine, Maharajgunj Medical Campus, \\ Institute of Medicine, Tribhuvan University, Kathmandu, Nepal \\ *Corresponding Author: ramesh.maharjan@mmc.tu.edu.np
}

\begin{abstract}
Emergency workers carry a huge responsibility in Covid 19 pandemic. Emergency staff have to provide prehospital care, early triage and management of patients with suspected or confirmed infection. More importantly, emergency care givers have to do everything to prevent emergency room from spread of the infection, to other patients. Access blocks following increasing flow of patients with suspicion of infection, causes overwhelming patients leading to delayed care and resource shortage to patients with chronic and acute diseases. This surge has put emergency workers at risk: risk from a potential contamination and risk from the effect of extended shifts work in the hospital. At this stage, the major challenges while performing emergency service has been managing triage, human resources, limited stock of personal protective equipment (PPE), proper management of infection prevention and limited laboratories for testing.
\end{abstract}

Due to lack of those requirements, certain guidelines and running the service without such guidelines making the emergency service in difficult situation during such crisis period.

Keywords: emergency response - Covid-19 - resource limited - TUTH, Nepal 


\section{INTRODUCTION}

Corona virus disease 2019 (COVID-19) is the illness associated with the novel corona virus known as severe acute respiratory syndrome corona virus 2 (SARS-CoV-2). The first case of COVID-19 was reported from Hubei Province of China on 31 December 2019 (Hui et al 2020). The cases continued to spread outside of the area and then across the world. Public Health Emergency of International Concern (PHEIC) was announced on 30th January 2020 and as a pandemic on 11 th March 2020 (WHO 2020). In Nepal, the first case was reported on 23rd January 2020, a 32-year old Nepali man returning from Wuhan (Shrestha et al 2020). The Government of Nepal announced suspension of all international flights followed by a country-wide full lockdown from 23rd March 2020 onwards and partial lock down till date (Pradhan 2020).

Infected persons with underlying comorbid conditions seem to have fetal outcome to COVID-19. Mortality rates also increase with age, as noted in data from the United States, as well as worldwide. This is related to the fact that persons over age 65 years, but especially to the fact that persons over age 85 years, are more likely to have a severe form of the illness (Chow et al 2020).

Emergency Medicine's role in this crisis is crucial. Emergency triage is the first barrier separating COVID and Non-COVID patients in the health care institutions. Furthermore, for COVID suspected and confirmed patient's emergency is the only place to decide: self-quarantine, discharge for home isolation, admission to dedicated COVID ward or ICU facility or dead body care if brought dead. Additionally, even if patients have only mild respiratory symptoms, they intuitively present in the Emergency room once they fear to have a serious disease. Therefore, emergency room needs to prepare fast and effectively to address the challenge of walk-in patients who might transmit the virus SARS- CoV-2 without displaying severe symptoms themselves. It is also responsibility of emergency care provider to protect vulnerable patient groups (immunosuppressed, chronic disease like diabetes, hypertension, older age) from transmission through asymptomatic COVID-19 patients (Chow et al 2020).In France, hospitals and emergency departments in particular try to adapt to the ever-increasing flow of patients with suspicion of infection that constitutes serious access blocks. Due to overcrowded departments and wards, patients with other chronic and acute disease may encounter delayed care (antibiotics, 
analgesics, thrombolysis etc.) and may suffer from resource shortage (Cao et al 2020), (Richardson et al 2009). This has led to unequivocal need of planning and guidance to surge capacity in emergency following COVID era. Furthermore, among different medical specialties, one survey has found Anesthesiology, Emergency physicians and Ophthalmologist are at greatest risk of contracting COVID-19 disease (Breazzano et al 2020). So it's mandatory to have utmost measures on infection prevention and control to halt iatrogenic harm.

The COVID-19 pandemic and global response to it have rapidly been changing and so its clinical management. It has to be acknowledged that even a day after submission of this article, this will become outdated. We choose nevertheless to initiate this study, in part, as a guide to all emergency physicians so that management of cases is properly executed and also to record experience of emergency physician in this unique period. The aims of the study was to provide a rigid guiding framework to emergency frontline staff for timely, efficient and effective response to minimize adverse event of COVID-19 pandemic and to provide strategies for human resource capacity building and management, safety and security, mental wellbeing during COVID-19 pandemic.

\section{METHODOLOGY}

PubMed, Cochrane Database of Systematic Reviews resources from 2012 to 2020 were accessed using the keywords emergency department, epidemic, pandemic, corona virus, SARS- CoV-2, and COVID-19. The websites of the WHO; the United States Centers for Disease Control and Prevention (CDC); and www.EMCrit.org were also accessed.

In Nepal, Ministry of Health and Population (www.mohp.gov. $n p$ ) is the leading authority in safeguarding public health. World Health Organization (WHO), Center for Disease Control (CDC) and peer-reviewed articles/publications are referenced by MoHP followed by publication of guidelines. It updates information about risk areas and recommendation for diagnostic and hygiene measures daily. All COVID-19 related existing Nepal's national policies, strategies, instructions, guidelines and evidences were reviewed while developing this plan. Some of the guidelines made by Nepal Ministry of Health and Population (MoHP) relevant to treating COVID-19 patients in emergency services which were reviewed while making this response plan are as follows :- 
Health sector Emergency Response Plan COVID-19, May 2020 (MoHP Nepal 2020),

Pocket book of Clinical Management Guideline (MoHP Nepal 2020), Interim guideline for Infection prevention and control measures, May 12, 2020 (MoHP Nepal 2020),

Guidelines for use of Personal Protective Equipment (PPE) (Personal protective equipment guidelines for health care workers at Tribhuvan University Teaching Hospital, Maharajgunj Medical Campus 2020), Health and Waste management guideline (MoHP Nepal 2014),Dead body Management guideline (MoHP Nepal 2020), Patient transfer Team (PTT) guidelines (MoHP Nepal 2020). Further international articles, were identified relating to COVID-19 which reported management of emergency patients during pandemic (Acharya et al 2020), (Testing PCR guidelines for COVID-19, TUTH 2020), (Josh 2020). Guidelines formed by two prestigious tertiary hospitals treating COVID-19 patients in Nepal: Tribhuvan University Teaching Hospital (TUTH), Patan Academy of Health Science (PAHS) were reviewed (Acharya et al 2020), (Testing PCR guidelines for COVID-19, TUTH 2020).

\section{RESULTS}

\section{Crucial management considerations}

\section{Prehospital care}

When individuals contact local emergency services, like 102(Nepal Ambulance Service), may ask callers if they are experiencing symptoms of a viral illness. This information is relayed to the responding provider so preparation can be made for an encounter with the patient, which includes donning the appropriate personal protective equipment (PPE) (Nepal Ambulance Service 2020).

Ambulance Driver during Patient Transport: (Richardson et al 2009) (MoHP Nepal 2020) have urged to maintain social distance, especially during patient transport, hand wash with soap and water/ hand rub- alcohol based solution. One must wear medical/surgical mask and face shield/ goggles during transporting patient. The need to wear gown if possible fluid resistant gown when transporting patient, wear hand gloves during patient contact and wash hand before and after gloves use. 
For patients requiring treatment of respiratory symptoms, extreme caution is advised. Aerosolizing procedures are high risk for transmission of COVID-19. An N95 mask, eye protection, and gown should be required by protocols. If possible, aerosolizing procedures should be performed outside of the ambulance prior to transport (Interim Guidance for Emergency Medical Services (EMS) Systems and 911 Public Safety Answering Points (PSAPs) for COVID-19 in the United States (CDC 2020a).

\section{Triage}

a. Main objective of triage during infectious disease outbreak is to separate infectious patients from others, to minimize cross contamination (Testing PCR guidelines for COVID-19, TUTH, 2020). For it, allocation of a separate, dedicated geographical area for triaging, fever clinic and admitting COVID-19 suspected, probable and confirmed cases should be done (Acharya et al 2020), (Testing PCR guidelines for COVID-19, TUTH, 2020). Triage process needs to be changed according to the evolving scenario. If one infected person presents to a busy emergency room triage area, there is a high likelihood of spreading the virus and potentially contaminating others. The $\mathrm{CDC}$ recommends placing ample touchless hand sanitizer stations, hand wash stations and easy-todispense boxes of face masks at entrances to the emergency room and hospital (Weingart 2020).

b. Since pre-notification systems exist in most of emergency services, prior notification can be done by pre-hospital care provider or referral health institution for suspected patient of COVID-19.

c. Create primary and secondary triage station that runs 24 hours a day with standard health desk equipped with full set PPE, supplies for screening and transportation facilities for referral (Josh Farkas 2020).

d. Screening - based on a standardized case definition (WHO 2020) have initiated to refer suspect cases for full investigation and laboratory confirmation of COVID-19. While screening look for Influenza like Illness (ILI) and Severe Acute Respiratory Illness (SARI) patients (Josh Farkas 2020).ILI- acute respiratory infection with fever $\geq 38^{\circ} \mathrm{c}$ and cough or severe acute respiratory illness. 
SARI - severe acute respiratory infection with fever $\geq 38^{\circ} \mathrm{c}$ and cough and requiring hospitalization.

e. Primary triage station is set at the first entry to the regular emergency department where triage officer will ask if the patients fulfill any criteria to meet for ILI or SARI. If the patient meets the criteria then he/she will be directed towards the fever clinic, if not then towards the regular emergency. Patients who are suspected of being infected need to have a mask applied promptly. Critically ill patients may have already been intubated, if not should be stabilized. Patients with intermediate symptoms may present on BiPAP or nebulizer treatments. All aerosolizing procedures require PPE for the receiving staff members in the Emergency (Josh Farkas 2020).

f. In fever clinic, a secondary triage officer will extensively try to detect, notify and refer ILI and SARI patients to designated COVID area where isolation and management is done or if treating physician is not convinced clinically, patient is sent to regular nonCovid emergency. Triage staff should be aware of the possibility that any patient coming through their door may have COVID-19. Stable patient may only require a small amount of supplemental oxygen via a nasal cannula. All patients should be kept at least 6 feet apart and remain with masks in place while their disposition is determined (MoHP Nepal 2020), (Josh Farkas 2020), (Standard Operating Procedure (SOP) for Triage of Suspected COVID-19 Patients in non-US Healthcare Settings: Early Identification and Prevention of Transmission during Triage CDC 2020b).

g. COVID19 suspected, probable and confirmed cases should be segregated and kept in separately dedicated geographical area to eliminate chance of transmission. It is better to have separate Emergency room, Inpatient ward, Operation theatre (OT) and monitoring Intensive Care Unit (ICU) for those patients. This service is separated from regular emergency service and separate from emergency department building in TUTH, Kathmandu, Nepal. This is recommended and followed by other major hospitals of Nepal (Testing PCR guidelines for COVID-19, TUTH 2020). 


\section{Critically ill patients}

may have already been intubated. Patients with intermediate symptoms may present on BiPAP or nebulizer treatments. Both are aerosolizing procedures that require PPE for the receiving staff members in the Emergency. Patient might be transitioned to a non-rebreather mask.

Personal Protective Equipment's (PPEs)- PPEs for Emergency Department: Hand Rub/ Hand wash - all health workers, patient, patient visitors should be encouraged to wash hand, wear mask before entering emergency department. For that, soap-water or alcohol-based hand rub should be available in entry and exit door (MoHP Nepal 2020), (PPE Guidelines for health care workers at TUTH 2020); Proper donning required: follow sequence $\rightarrow$ Cap (with full hair covered) $\rightarrow$ Fluid resistant gown $\rightarrow$ N95 or Medical/surgical mask (acceptable) $\rightarrow$ Face shield: when in close contact of patient $<2 \mathrm{~m} \rightarrow$ Gloves (loose gloves acceptable)(MoHP Nepal 2020),

(PPE Guidelines for health care workers at TUTH 2020): Resuscitation area: where aerosol generation procedures are done medical/surgical mask has to be replaced by N95 respirator and goggles/face shield must be worn during procedure (MoHP Nepal 2020), (PPE Guidelines for health care workers at TUTH 2020); Aerosol generating procedure done in emergency room- Cardiopulmonary Resuscitation (CPR), Intubation, Manual ventilation, Tracheostomy procedures, open suctioning, non- invasive ventilation (BiPAP, CPAP), Nebulization therapy, sputum induction, obtaining nasopharyngeal or oropharyngeal swab, Otorhinolaryngology procedure, dental procedures, upper GI endoscopy, bronchoscopy) (MoHP Nepal 2020), (PPE Guidelines for health care workers at TUTH 2020).

Doffing at designated area ONLY. Doffing of personal protective equipment (PPE) is often the highest-risk procedure during the patientphysician interaction, in terms of spread of SARS- CoV-2. - Preferably ONCE per shift (MoHP Nepal 2020), (PPE Guidelines for health care workers at TUTH 2020). Procedure for Doffing is as follows - Clean gloves with alcohol-based hand sanitizer; Remove gown and gloves; Pull the gown forward to tear the gown away from the body; Roll gown inside out and away from the body, bending forward, creating a"glove/ gown ball". Clean hands with alcohol-based hand sanitizer; Remove 
face shield; Don't touch the front of the shield, which may be dirty; Remove by touching the band of the face shield; Clean hands with alcohol-based hand sanitizer (MoHP Nepal 2020), (PPE Guidelines for health care workers at TUTH 2020), (Acharya et al 2020).

Allied health care workers: laundry/waste management/ housekeeping/ mortuary staff: (MoHP Nepal 2020), (PPE Guidelines for health care workers at TUTH 2020): Hand wash with soap and water/ Hand rubAlcohol based solution; Medical/surgical mask; Gloves: only during patient care and handling materials. Hand wash before and after gloves use; Gown: Fluid resistant gown and Boot required all the times.

\section{Differential diagnosis}

The initial stages of COVID-19 infection are nonspecific and include symptoms typical of multiple respiratory illnesses, viral and otherwise(Cascella et al 2020), (COVID-19 2020).

Viral infections (which may occur simultaneously with COVID-19) in the differential diagnosis include the following (Cascella et al 2020), (COVID-19 2020)-Influenza, Parainfluenza, Human metapneumovirus, Human rhinovirus, Adenovirus and Respiratory syncytial virus. Bacterial infections of differential diagnosis include Haemophilus influenzae, Streptococcus pneumoniae and Moraxella catarrhalis. Atypical pneumonia in the differential diagnosis includesLegionellosis, Mycoplasma pneumoniae pneumoniae.

Approach to Covid emergency department patients getting isolated/ admitted to the hospital: Wear proper PPE - as described above; High risk group - Travel history or contact history in community transmission area, Patient has been close contact $<6 \mathrm{ft}$ with known positive or suspected patient, Symptoms-Fever (83-99\%), Cough (59-82\%), Fatigue (44-70\%), Anorexia (40-84\%), Shortness of breath (31-40\%), Myalgia (11-35\%)(WHO 2020).

Non-specific symptoms: Diarrhea, Anosmia, Dysgeusia. Patients are reporting onset of symptoms over a period of week, with rapid progression to respiratory distress around day 8 (Huang C et al. 2020).

Severe conditions- Septic shock, Diabetic ketoacidosis, Acute Kidney Injury (AKI), acute cardiac injury and Dysrhythmias (Chen et al 2020)- Physical examination- Fever, Tachycardia, Tachypnea, Hypoxia 
in pulse oximeter in initial findings (Wu et al 2020), Crepts (Pneumonia, Acute Respiratory Distress Syndrome (ARDS).

Diagnostic tests: Nasopharyngeal and Oropharyngeal swab for reversetranscription polymerase chain reaction (RT-PCR) assay.

Priority testing based on patient symptom, contact history, travel history, health care worker, hospitalized patients, older age, underlying non communicable disease (diabetes, hypertension, cardiac disease, chronic lung disease, cerebrovascular disease, chronic kidney disease, cancer, immunosuppression) (WHO 2020).RT-PCR positivity was highest in Broncho alveolar lavage specimens (93\%), followed by sputum (72\%), nasal swab (63\%), and pharyngeal swab (32\%) (Sethuraman et al 2020) (Wang et al 2020).

For prognosis - leukopenia, leukocytosis, elevated Liver enzymes, Creactive protein, Lactate dehydrogenase , Ferritin (21). D-dimer remains a debatable test, it has been found to be higher in patients with ARDS and death (Chen et al 2020).

Imaging-Chest radiography may show peri-bronchial thickening; Chest computed tomography (CT) scanning- supports more definitive diagnosis (Li Y, Xia L. 2020) - shows ground-glass opacities, usually in lower lobesCOVID-19 disease severity classification according to WHO Clinical Management interim guidance 27 May 2020 (WHO 2020).

Mild disease (No pneumonia) -Symptomatic patients meeting the case definition for COVID-19 without evidence of viral pneumonia or hypoxia. Treatment of Mild disease (No pneumonia): Symptomatic treatment such as antipyretics for fever and pain, adequate nutrition and appropriate rehydration and Antibiotic is not recommended

\section{Moderate disease (Pneumonia)}

Adolescent or adult: Clinical signs of pneumonia (fever, cough, dyspnoea, fast breathing) but no signs of severe pneumonia, including $\mathrm{SpO} 2 \geq 90 \%$ on room air.

Child: Clinical signs of non-severe pneumonia (cough or difficulty breathing + fast breathing and/or chest indrawing) and no signs of severe pneumonia. 
Treatment of Moderate disease (Pneumonia): Patient is isolated to contain virus transmission; Isolation in hospital is preferred with close follow-up and Antibiotics should not be prescribed unless there is clinical suspicion of a bacterial infection

Severe disease (Severe Pneumonia): Adolescent or adult: clinical signs of pneumonia (fever, cough, dyspnoea, fast breathing) plus one of the following: a, Respiratory rate $>30$ breaths/min; b, Severe respiratory distress; or c, $\mathrm{SpO} 2<90 \%$ on room air. Child: Clinical signs of pneumonia (cough or difficulty in breathing) and at least one of the following additional sign such as: Central cyanosis or $\mathrm{SpO} 2<90 \%$; severe respiratory distress (e.g. fast breathing and inability to breast feed or drink, lethargy or unconsciousness, or convulsions (Fast breathing (in breaths/min) $:<2$ months: $\geq 60 ; 2-11$ months: $\geq 50 ; 1-5$ years: $\geq 40)$.

Treatment of Severe disease (Severe Pneumonia): The setup should be equipped with pulse oximeters, functioning oxygen systems and disposable, single-use, oxygen-delivering interfaces (nasal cannula, Venturi mask, and mask with reservoir bag).There is a provision of immediate administration of supplemental oxygen therapy to any patient with emergency signs and to any patient without emergency signs and $\mathrm{SpO} 2<90 \%$. Health care of Cautious fluid management, watch for fluid overload and closely monitor patients for signs of clinical deterioration, such as rapidly progressive respiratory failure and shock. Monitor signs or symptoms suggestive of venous or arterial thromboembolism, such as stroke, deep venous thrombosis, pulmonary embolism or acute coronary syndrome.

Critical Disease: Acute Respiratory Distress Syndrome (ARDS) appear with (i) Onset within 1 week of a known clinical insult (i.e. pneumonia) or new or worsening respiratory symptoms and (ii) Chest imaging: radiograph, CT scan, or lung ultrasound, bilateral opacities, not fully explained by volume overload, lobar or lung-collapse, or nodules.

\section{Origin of pulmonary}

It infiltrates Respiratory failure not fully explained by cardiac failure or fluid overload and need objective assessment (e.g. echocardiography) to exclude hydrostatic and cause of infiltrates/edema if no risk factor present.

\section{Oxygenation impairment in adults}

(i) Mild ARDS: $200 \mathrm{mmHg}<\mathrm{PaO} 2 / \mathrm{FiO} 2 \mathrm{a} \leq 300 \mathrm{mmHg}$ (with PEEP or $\mathrm{CPAP} \geq 5 \mathrm{cmH} 2 \mathrm{O}$ ); (ii) moderate ARDS: $100 \mathrm{mmHg}<\mathrm{PaO} 2 / \mathrm{FiO} 2 \leq 200$ 
mmHg (with PEEP $\geq 5$ cmH2O) and (iii) Severe ARDS: PaO2/FiO2 $\leq 100$ $\mathrm{mmHg}$ (with $\mathrm{PEEP} \geq 5 \mathrm{cmH} 2 \mathrm{O}$ ).

Treatment of Acute Respiratory Distress Syndrome (ARDS): Mild ARDS - a trial of HFNO, non-invasive ventilation- CPAP, BiPAP may be used.

Remarks- need to remember are intubation should not be delayed if the patient acutely deteriorates or does not improve after a short trial and all staff entering for NIV or HFNO should wear appropriate PPE and adequate environmental ventilation is ensured.

Who needs ARDS intubation and invasive mechanical ventilation: Endotracheal intubation should be performed by a trained and experienced provider using airborne precautions.

Remarks-Avoid bag-valve mask ventilation to reduce exposure to aerosols; Attach viral filters in between mask and bag of AMBU bag to prevent expiratory air transmission; Use aerosol protection if available - aerosol block, Lucite cube, garbage bag; Rapid Sequence Intubation (RSI) with high-dose paralytic, ketamine is preferred induction but if patient is not compliant with preoxygenation, perform immediate ketamine dissociation for Delayed sequence Intubation; Highest-Level Physician should do the Intubation; Use video laryngoscope if available with new bougies to maximize 1-attempt success; Visualize black line of ETT at level of cords to avoid having to auscultate for depth; Cuff fully inflated and viral filter on the ETT prior to bagging/hooking to ventilator; lower tidal volumes (4-8 $\mathrm{mL} / \mathrm{kg}$ predicted body weight; In adult patients with severe ARDS ( $\mathrm{PaO} 2 /$ FiO2<150) prone ventilation for 12-16 hours per day is recommended. Use a conservative fluid management strategy for ARDS patients without tissue hypoperfusion and fluid responsiveness; after the patient has been intubated, admission to the hospital will be required.

ARDS in whom lung protective ventilation strategy fails to achieve adequate oxygenation and ventilation: Extracorporeal membrane oxygenation (ECMO), consider referral of patients who have refractory hypoxaemia (e.g. including a ratio $[\mathrm{PaO} 2 / \mathrm{FiO} 2]$ of $<50 \mathrm{mmHg}$ for 3 hours, a $\mathrm{PaO} 2: \mathrm{FiO} 2 \mathrm{of}<80 \mathrm{mmHg}$ for $>6$ hours) despite lung protective ventilation. Sepsis: In such situation (adult) acute life-threatening organ dysfunction caused by a dysregulated host response to suspected or proven infection. 
Signs of organ dysfunction include: Altered mental status, Difficult or fast breathing, Low oxygen saturation, Reduced urine output, Fast heart rate, weak pulse, cold extremities or Low blood pressure, Skin mottling,

Laboratory evidence: Coagulopathy, Thrombocytopenia, Acidosis, High Lactate, or hyperbilirubinemia. In case of children; Suspected or proven infection and $\geq 2$ age-based SIRS criteria of which one must be abnormal temperature or white blood cell count.

Septic shock: Adults: persistent hypotension despite volume resuscitation, requiring vasopressors to maintain $\mathrm{MAP} \geq 65 \mathrm{mmHg}$ and serum lactate level $>2 \mathrm{mmol} / \mathrm{L}$.

Children: Any hypotension $(\mathrm{SBP}<5$ th centile or $>2 \mathrm{SD}$ below normal for age) and two of the following: i. Altered mental status; ii.Bradycardia or tachycardia ( $\mathrm{HR}<90 \mathrm{bpm}$ or $>160 \mathrm{bpm}$ in infants and heart rate $<70 \mathrm{bpm}$ or $>150 \mathrm{bpm}$ in children); iii. Prolonged capillary refill $(>2 \mathrm{sec})$ or weak pulse; iv. Fast breathing; v. Mottled or cool skin or petechial or purpuric rash; vi. High lactate; vii. Reduced urine output; viii. Hyperthermia or hypothermia

Treatment of Septic shock: Includes early recognition and the following treatments to be done immediately, within 1 hour of recognition: antimicrobial therapy, and initiation of fluid bolus and vasopressors for hypotension; In resuscitation for septic shock in adults, give $250-500 \mathrm{~mL}$ crystalloid fluid as rapid bolus in first 15-30 minutes; In resuscitation for septic shock in children, give $10-20 \mathrm{~mL} / \mathrm{kg}$ crystalloid fluid as a bolus in the first 30-60 minutes; If there is no response to fluid loading or signs of volume overload appear (e.g. jugular venous distension, crackles on lung auscultation, pulmonary oedema on imaging, or hepatomegaly), then reduce or discontinue fluid administration. In adults, administer vasopressors when shock persists during or after fluid resuscitation. The initial blood pressure target is MAP $\geq 65 \mathrm{mmHg}$ in adults and improvement of markers of perfusion

Prevention of complications in hospitalized and critically ill patients with COVID19:

Monitor signs or symptoms suggestive of thromboembolism, such as stroke, deep venous thrombosis, pulmonary embolism or acute coronary syndrome and use pharmacological prophylaxis, such as low molecular weight heparin (such as enoxaparin). 
Some drugs such as antivirals, immunomodulators and other adjunctive therapies are not recommended to administer treatment or prophylaxis for COVID-19, outside of the context of clinical trials (WHO 4 July 2020): Chloroquine and hydroxychloroquine, azithromycin; Antivirals- Lopinavir/ritonavir; Remdesivir, Umifenovir Favipiravir; Immunomodulators- Tocilizumab, Interferon- $\beta-1 \mathrm{a}$; Plasma therapy; Systemic corticosteroids for treatment of viral pneumonia; ACEI, ARB to date, there are no studies that can substantiate this, and it is generally advised to continue these medications unless there are other reasons to stop these (e.g. hyperkalaemia, hypotension or acute deterioration in renal function).Discontinue transmission-based precautions (including isolation) and release from the COVID-19 care pathway as follows: For symptomatic patients: 10 days after symptom onset, plus at least 3 days without symptoms (without fever and respiratory symptoms).

For asymptomatic patients: 10 days after test positive

Reporting of death is recommended to use COVID-19 instead of corona virus during death certification as there are 6 subtypes of corona virus. A death due to COVID-19 is defined for surveillance purposes as a death resulting from a clinically compatible illness, in a probable or confirmed COVID-19 case, unless there is a clear alternative cause of death that cannot be related to COVID-19 disease (e.g. trauma). There should be no period of complete recovery from COVID-19 between illness and death.

Cleaning and disinfection practices: Ethyl alcohol (70\%) - Reusable dedicated equipment (e.g. stethoscope, thermometers etc.) (MoHP Nepal 2020); $0.5 \%$ Chlorine solution (e.g. sodium hypochlorite or equivalent disinfectant)- Infrastructures - floors, table tops, furniture, bed and handrails, door handles, light switches, tap knobs, toilet bowls.Reusablegoggles/visors, boots, utility gloves, heavy duty gloves, plastic aprons (MoHP Nepal 2020); 0.05\% Chlorine solution: Laundry- soiled laundry/ linen, scrub, water proof gown.

Procedure - Machine wash at 60-90॰C (140-194॰ F) with laundry detergent OR in absence of machine Soak laundry, linen in hot water and soap in a large bucket/drum, use a stick to stir, avoid splashing then empty the bucket of water, then soak linens in $0.05 \%$ chlorine for 30 minutes, then rinse and dry in sunlight (MoHP Nepal 2020).

Waste disposable of PPE- multiple bucket approach for separation of disposable items from reusable items, like disposable waste; glasses/eye shields; gowns (laundry items) in 3 separate buckets (MoHP Nepal 2020). 
Surge capacity (MoHP Nepal 2020): Restrict number of HCW in ERreduces exposure, availability of backup manpower if cluster of staffs gets quarantined, minimizes use of PPE; Use of hotline emergency number and telemedicine to minimize hospital visits by care seekers; Restrict visitors in ER by using visitor pass - encourage phone, online social media chats and messaging; Minimize use of PPE-use of glass or plastic windows where patient first present (triage/fever clinic/ reception) and reprocessing N95 using UV radiation lamp, vapor, hydrogen peroxide, ethylene oxide.

Human resource capacity building and management (MoHP Nepal 2020): Recognize, pre-position, instruct/teach, drill medical personnel, nurses, paramedics, and other personnel regarding hand hygiene, respiratory hygiene, proper donning and doffing of PPE, waste disposition, transportation of patient, care of diseased body. A benefit package should be developed for the frontline health workers and support staff and need an implementation of life and health insurance of the health workers as per 2020 budget announcement.

Safety and security of the frontline staff (MoHP Nepal 2020): Purchase and stockpile personal protective equipment including water resistant own, medical/surgical mask for standard precaution; gloves, face shield, googles for droplet and contact precaution; and N95 mask for aerosol generating procedures as per protocol; Security system should be strengthened with adequate security personnel; Any stigma and discrimination, violence and any forms of harassment towards emergency health workers engaged in the COVID-19 response process should be monitored and take necessary measures to avoid them and in case of occurrence necessary legal actions should be taken with ZERO tolerance to violence; Hotel accommodation and food for Frontliners working in COVID emergency should be managed.

Logistics team should be formed(MoHP Nepal 2020): There is a need to communicate and convene meeting with key stakeholders; assess critical needs; develop quick response plan based on the critical needs; ensure effective supply chain management; Collect information and report to relevant authorities; Adequate supply of necessary PPE and other commodities such as oxygen, blood gas analyzer, emergency essential drugs and other essential items should be estimated and ensured through hospital administration; 24/7 internet services should be established

\section{Mental health service and psychosocial counselling}

Psychiatric counselling and support should be provided to health care workers through appropriate medium such as group counselling Apps (MoHP Nepal 2020). 


\section{Risk communication messages}

Standard messages in local languages developed by NHEICC referencing WHO, MoHP guidelines and peer-reviewed articles/publications need to be posted. Some of them might be regarding physical distancing, hand hygiene, respiratory hygiene (MoHP Nepal 2020).

\section{CONCLUSION}

The single best way to save the most people and reduce morbidity and mortality is to be proactive and not reactive. Things needs to be done differently and above recommendations should be implemented sooner the better in emergency services of different hospitals. We need to learn from other countries' mistakes and perform early testing and strict isolation which is what epidemiologists recommend to control infectious outbreaks. However, Emergency Frontliners are always in high risk of unknowingly contact with Covid-19 cases in Emergency Service. Hence, it is mandatory and better way of dealing such cases with above guidelines from PreHospital to In-Hospital emergency care system. We must follow the principle of "Do No Harm" as usual in such period of Covid 19 Pandemic Crisis in Nepal. Please rephrase sentence as message is not clear

\section{DECLARATION}

No conflict of interest from all authors.

\section{REFERENCES}

Acharya, S., Maharjan, K. (2020). Triaging in COVID-19 at Patan Hospital, Nepal. J Patan Acad Heal Sci, 7(1): 77-79. https://www.jpahs.edu. np/index.php/JPAHS/article/view/293

Breazzano, M.P., Shen, J., Abdelhakim, A.H., Glass, L.R.D., Horowitz, J.D., et al. (2020). Resident physician exposure to novel coronavirus (2019-nCoV, SARS-CoV-2) within New York City during exponential phase of COVID-19 pandemic: Report of the New York City Residency Program Directors COVID-19 Research Group. medRxiv https://www.medrxiv.org/content/10.1101/2020.0 4.23.20074310v1

Cao, Y., Li Q, Chen, J., X., Miao C., Yang, H., et al. (2020). Hospital Emergency Management Plan During the COVID-19 Epidemic. Academic Emergency Medicine. Blackwell Publishing Inc.; Vol. 27, p. 309-311. https://www.ncbi.nlm.nih.gov/pmc/articles/ PMC7159322/ 
Cascella M, Rajnik M, Cuomo A, Dulebohn SC \& Di Napoli R. (2020). Features, Evaluation and Treatment Coronavirus (COVID-19). StatPearls. Stat Pearls Publishing. http://www.ncbi.nlm.nih.gov/ pubmed/32150360

CDC (2020a). Interim Guidance for Emergency Medical Services (EMS) Systems and 911 Public Safety Answering Points (PSAPs) for COVID-19 in the United States. https://www.cdc.gov/ coronavirus/2019-ncov/hcp/guidance-for-ems.html

CDC. (2020b). Standard operating procedure (SOP) for triage of suspected COVID-19 patients in non-US healthcare settings: Early identification and prevention of transmission during triage. https:// www.cdc.gov/coronavirus/2019-ncov/hcp/non-us-settings/soptriage-prevent-transmission.html

Chen, N., Zhou, M., Dong, X., Gong, F. \& Han, Y. (2020).Epidemiological and clinical characteristics of 99 cases of 2019 novel coronavirus pneumonia in Wuhan, China: a descriptive study. Lancet, 395(10223):507-13. https://doi.org/10.1016/

Chen, T., Wu, D., Chen, H., Yan, W. \& Yang, D. (2020). Clinical characteristics of 113 deceased patients with coronavirus disease 2019: Retrospective study. BMJ, 26: 368. https://www.bmj.com/ content/368/bmj.m1091.

Chow, N., Fleming-Dutra K, Gierke, R., Hall, A., Hughes, M., Pilishvili, T., et al. (2020). CDC COVID-19 Response Team. Preliminary Estimates of the Prevalence of Selected Underlying Health Conditions Among Patients with Coronavirus Disease 2019 United States. Morbidity and Mortality Weekly Report. Department of Health and Human Services Vol. 69, p. 382-386. https://www. cdc.gov/mmwr/volumes/69/wr/mm6913e2.htm

Huang, C., Wang, Y., Li X, Zhao, J. \& Hu, Y. (2020). Clinical features of patients infected with 2019 novel coronavirus in Wuhan, China. Lancet, 395(10223): 497-506. http://www.thelancet.com/article/ S0140673620301835/fulltext

Hui D.S., I Azhar E, Madani T.A., Ntoumi, F., Kock, R., Dar, O., et al. (2020). The latest 2019 novel coronavirus outbreak in Wuhan, China. International Journal of Infectious Diseases. https://mohit. pure.elsevier.com/en/publications/the-continuing-2019-ncovepidemic-threat-of-novel-coronaviruses. (Accessed: 01.02.2020)

Josh Farkas (2020). COVID-19 - Emcrit Project. https:/emcrit.org/ibcc/ COVID19/ 
Li, Y., Xia L.(2020). Coronavirus disease 2019 (COVID-19): Role of chest CT in diagnosis and management. American Journal of Roentgenol, 214(6):1280-1286. www.ajronline.org

Ministry of Health and Population Nepal. (2020). Health Sector Emergency Response COVID-19. https://drive.google.com/file/d/1Jrg02HTqNq8KkUESCn e35GreQOL199h/view

Ministry of Health and Population Nepal (2020). Pocket book climical mgmt. https://drive.google.com/file/d/1 aa4R7r7AgwY83PjDqw_ BwlsWk4p6mTkZ/view

Ministry of Health and Population Nepal. (2020). Pocket book Infection Prevention Control HC 31-01-2077. https://drive.google.com/file/ d/1qCKEq0CqDeGQTyKU_EwVkyrf5X9M80X8/view

Ministry of Health and Population Nepal (2014). Health Care Waste Management Guideline. Government of Nepal, Ministry of Health and Population.

Ministry of Health and Population Nepal. (April 8 2020). Dead body management protocol. https://drive.google.com/ file/d/1ENGIDUd_qlxuoTp44VTsMiileaC0q0LW/view. Assessed: 08.04.2020

Ministry of Health and Population Nepal (2020). COVID-19 Patient transport team (PTT) guideline. https://drive.google.com/file/ d/1GLzSSLS_z8mkZTeXoD3uaB6PcqbefJT/view

Nepal Ambulance Service (2020). Saving lives along the way. https://www. nepalambulanceservice.org/

Personal Protective Equipment. (2020). Guidelines for health care workers at Tribhuvan University Teaching Hospital, Maharajgunj Medical Campus.

Pradhan, T.R., (2020, March 23). Nepal goes under lockdown for a week starting 6am Tuesday. The Kathmandu Post. https://kathmandupost. com/national/2020/03/23/nepal-goes-under-lockdown-for-a-weekstarting-6am-tuesday.

Richardson, D.B. \& Mountain, D. (2009) Myths versus facts in emergency department overcrowding and hospital access block. Medical Journal, 190(7): 369-374.

Sethuraman, N., Jeremiah, S.S. \& Ryo, A. (2020). Interpreting Diagnostic Tests for SARS-CoV-2. Journal of the American Medical Association (JAMA), 323: 2249-2251. https://jamanetwork.com/

Shrestha, R., Shrestha, S. \& Khanal, P., (2020). Nepal's first case of COVID-19 and public health response. Journal of Travel Med. 
TUTH. (2020). Testing PCR guidelines for COVID-19, Tribhuvan University Teaching Hospital, Maharajgunj Medical Campus.

Wang, W., Xu, Y., Gao, R., Lu, R. \& Han, K. (2020). Detection of SARSCoV-2 in Different Types of Clinical Specimens. Journal of the American Medical Association (JAMA), 323: 1843-1844. https:// jamanetwork.com/

Weingart, S. (2020). COVID Airway Management Thoughts . Emcrit.org https://i0.wp.com/emcrit.org/wp-

WHO (2020). Clinical management of COVID -19- Interim guidance. World Health Organization.

WHO (2020). Coronavirus disease (COVID-19) Pandemic. Vol. 43. https:// www.who.int/emergencies/diseases/novel-coronavirus-2019

Wu, Z., McGoogan, J.M. (2020). Characteristics of and Important Lessons from the Coronavirus Disease 2019 (COVID-19) Outbreak in China: Summary of a Report of 72314 Cases from the Chinese Center for Disease Control and Prevention. Vol. 323, Journal of the American Medical Association (JAMA), 323: 1239-1242. American Medical Association. https://jamanetwork.com/. 